\title{
Die Österreichische Schule als Gegenprogramm zur Standardökonomik: Replik zum Kommentar von Ansgar Belke
}

\author{
Karl-Friedrich Israel · Jörg Guido Hülsmann
}

Online publiziert: 27. Februar 2019

(C) List-Gesellschaft e.V. 2019

Wir stimmen Ansgar Belke zu, dass es viele inhaltliche und auch so manche methodische Überschneidungspunkte zwischen der Österreichischen Schule (ÖS) und der Standardökonomik (SÖ) gibt. Das haben wir in unserem Referat auch unterstrichen. Unsere Aufgabe bestand aber darin, darzulegen, inwiefern die ÖS ein alternatives Forschungsprogramm zu fast allen anderen Richtungen der heutigen Volkswirtschaftslehre darstellt und worin wir die wissenschaftliche und praktische Bedeutung dieser Alternative sehen.

Der Ausdruck Gegenprogramm stammt nicht aus unserer Feder, sondern wurde vorgegeben. Er erschien uns unproblematisch, aber Belkes Kommentar zeigt, dass er auch in einem engeren Sinne, den wir durchaus nicht beabsichtigt hatten, aufgefasst werden kann. Wir meinen nicht, dass die Arbeiten der ÖS das Ziel verfolgen, andere Denkrichtungen zu widerlegen oder zu diskreditieren. Der Zweck besteht vielmehr in der Erklärung der Wirklichkeit, und es wäre daher vielleicht klarer gewesen, von einem „konkurrierenden“ Forschungsprogramm innerhalb des von Belke genannten „Wettbewerbes der Denkschulen“ zu sprechen.

Belke weist immer wieder darauf hin, dass wichtige Positionen der Österreichischen Schule (ÖS) bereits in der Standardökonomik (SÖ) oder zumindest im interventionskritischen Teil des „Mainstreams“ integriert seien. Dies ist oftmals korrekt.

This reply refers to the commentary article available online at https://doi.org/10.1007/s41025-01900138-3.

\section{K.-F. Israel}

Institut für Wirtschaftspolitik, Universität Leipzig, Grimmaische Straße 12, 04109 Leipzig,

Deutschland

E-Mail: karl-friedrich.israel@uni-leipzig.de

J. G. Hülsmann $(\bowtie)$

Faculté de droit, d'économie et de gestion, Université d'Angers, 13, allée François

Mitterrand, 49036 Angers, Frankreich

E-Mail: guido.hulsmann@univ-angers.fr 
Wir halten selbstverständlich nicht alle der in unserem Aufsatz ausgeführten Aspekte für Alleinstellungsmerkmale der ÖS. Gerade im Hinblick auf die methodologischen Grundlagen geht Belke jedoch einen Schritt zu weit.

Dies wird insbesondere in den langen Ausführungen zur Lucas-Kritik deutlich. In unserem Beitrag haben wir eine Neuinterpretation der Lucas-Kritik angedeutet, „nicht als empirische Hypothese, sondern als eine grundsätzliche methodologische Einschränkung der Sozialwissenschaften [...] als ein warnender Hinweis, der a priori Gültigkeit beansprucht. “ ${ }^{1}$ Belke erwidert: „Dies ist jedoch [...] eindeutig keine neue Interpretation der Lucas-Kritik, sondern gibt nur die von Lucas (1976) selbst vorgebrachte Verallgemeinerung wieder. Stattdessen tun Israel und Hülsmann so, als ob die ÖS die Lucas-Kritik von einer angeblichen Verengung auf die Kritik an den großen keynesianischen Modellen befreien könnte. Hier wird ein unnötiger ,Strohmann“ aufgebaut.“

Einspruch! Lucas selbst hat sein Argument niemals in dieser verallgemeinerten Form präsentiert. Er ist davon ausgegangen, dass es grundsätzlich stabile empirische Beziehungen zwischen politisch relevanten ökonomischen Variablen gäbe, und dass diese auf Basis empirischer Untersuchungen identifiziert werden müssten. Die Lucas-Kritik gelte also nicht a priori für jedes quantitative ökonomische Modell. In der Einleitung zu seinen Studies in Business Cycle Theory hat Lucas dies deutlich formuliert:

I view "Econometric Policy Evaluation" (Lucas 1976) as a contribution to this important question of the relation of theory to econometric work, but sometimes it is read as though it resolved the issue much more decisively than could ever be the case. The paper stressed the importance of identifying structural parameters that are invariant under the kinds of policy changes one is interested in evaluating; and in all of the paper's examples, only the parameters describing "tastes" and "technology" were treated as having this property. This presumption seems a sound one to me, but it must be defended on empirical, not logical grounds, and the nature of such a defense presumably would vary with the particular application one has in mind. (Lucas 1981, SS. 11-12, unsere Hervorhebung)

Nach unserem Dafürhalten beschränkt sich die Standardinterpretation der LucasKritik heutzutage auf die Einsicht, dass gewisse theoretische Erwägungen unabdingbar seien, um relevante Schlussfolgerungen aus ökonometrischen Studien zu ziehen. So antwortete Lucas in einem Interview mit Howard Vane und Brian Snowdon auf die Frage: „How important do you think the ,Lucas critique“ has been?“", mit den folgenden Worten:

I think it has been tremendously important, but it is fading. It used to be that you could hold that up, like a cross to a vampire, and defeat people simply by saying "Lucas critique". People have gotten tired of that and I think that is fair enough. If you want to criticize work effectively you have to get into it and criticize its

\footnotetext{
${ }^{1}$ An anderer Stelle wurde diese Idee ausführlicher besprochen (Israel 2015, SS. 134-139). Zu diesem Thema siehe auch die von Belke selbst angeführte Analyse von Basse (2006).
} 
details. But I think it is basic that you can't get economic conclusions without putting in some economic theories, some economic structure. (Snowdon und Vane 1998, S. 128)

Auch wenn diese Einsicht überaus wichtig ist, entspricht sie nur einem Teilaspekt der von uns vorgeschlagenen Interpretation der Lucas-Kritik. ${ }^{2}$

Belke erklärt, dass „,der Lucas-Artikel einer der einflussreichsten makroökonomischen Beiträge überhaupt" sei. Das ist unbestreitbar. Aber es beweist keinesfalls, dass unsere Diagnose über die große Bedeutung von empirischen Prognosen für die wissenschaftliche Erklärung ökonomischer Phänomene und als methodologisches Leitmotiv in weiten Teilen der modernen Ökonomik ,,absurd“ sei. Ganz im Gegenteil. Die gängige Interpretation der Lucas-Kritik als empirische Hypothese ist sehr wohl mit diesem methodologischen Leitmotiv vereinbar. ${ }^{3}$

Wie wir in unserem Hauptbeitrag erwähnt haben, werden die modernen DSGEModelle oft als eine Lösung für die Lucas-Kritik angeboten. Auch dies zeigt, dass sie nicht als grundsätzliches Problem wahrgenommen wird, sondern aus Sicht vieler moderner Ökonomen nur in speziellen Fällen von Bedeutung ist.

Nun wird die kühne These, dass die Lucas-Kritik mit den DSGE-Modellen überwunden wurde, nicht von allen „Mainstream“-Ökonomen geteilt, wie Belke richtig ausführt. Insbesondere nach der großen Rezession von 2007, erhoben sich gegen diesen Modellansatz auch vermehrt kritische Stimmen aus dem „Mainstream“. Romer (2016) hat dabei für besonderes Aufsehen gesorgt. Er bezieht sich in seiner Kritik der modernen Makroökonomik explizit auf den DSGE-Ansatz, gerade weil er ihn für repräsentativ hält. ${ }^{4}$ Es ist keinesfalls so, wie Belke behauptet, dass „nur eine relativ kleine, hoch spezialisierte makroökonomisch orientierte Community" an und mit diesen Modellen arbeitet. Sie sind nicht „nur eine ,Strohpuppe“ als ,Whipping boy ' der Heterodoxen." Sie werden stattdessen sehr oft als Zugpferde der modernen makroökonomischen Forschung angesehen.

Es gibt DSGE-Modelle in ganz verschiedenen Ausführungen, von den frühen Modellen der realen Konjunkturzyklen mit Geldneutralität (real business cycle models), zu den modernen neukeynesianischen Modellen mit financial fricitons und realen Effekten der Geldpolitik. Sie werden in unterschiedlichen Varianten in allen großen Zentralbanken und anderen Institutionen zur Analyse von Geldpolitik herangezogen,

\footnotetext{
2 Für eine sehr lesenswerte Übersicht aus der sehr deutlich hervorgeht, dass die Lucas-Kritik als eine empirische Hypothese verstanden und interpretiert wurde, siehe Cruccolini (2010, Kap. 3).

3 In unserem Hauptbeitrag haben wir die Ursprünge dieses methodologischen Prinzips in aller Kürze besprochen. Hier sei noch einmal erwähnt, dass es in keiner Arbeit so deutlich hervorgehoben wurde wie in Friedmans The Methodology of Positive Economics (Friedman 1953). Dieser Artikel wurde zurecht als der einflussreichste Beitrag zur Methodologie der Wirtschaftswissenschaften im 20. Jahrhundert bezeichnet (Hausman 2008, S. 33).

4 Hier könnte man u.a. auch Stiglitz $(2011,2017)$ oder den eher journalistischen Beitrag von Krugman (2009) anführen, die sich ebenfalls in ihrer Grundsatzkritik auf die DSGE-Modelle beziehen. Dieses Vorgehen ist berechtigt, da der DSGE Ansatz tatsächlich von größter Bedeutung in der modernen Makroökonomik ist. Deshalb ist er nach der letzten Finanzkrise verschärft in die Kritik geraten. Für eine kürzlich erschienene Verteidigung des DSGE-Ansatzes gegen einige der vorgebrachten Kritiken, siehe Lindé (2018). Außerdem sei auch auf den Artikel zur (Mainstream-)Makroökonomik in diesem Sonderheft des List Forums verwiesen. Auch Bachmann (2019) hebt den DSGE-Ansatz hervor.
} 
und sind Bestandteil jedes Graduierten-Kurrikulums an namhaften Universitäten auf der ganzen Welt. ${ }^{5}$

Belke moniert, ,dass Kritik an DSGE-Modellen weder notwendig, noch hinreichend für fundierte Kritik am [...] nicht hinreichend differenzierten ,Mainstream “ ist.“ Dabei verkennt er zum einen, dass sie von vielen führenden Vertretern des Fachs als charakteristischer Bestandteil des „Mainstreams“ angesehen werden, und zum anderen, dass wir sie lediglich als Beispiele in eine breitere Literatur einbetten, von der sich die ÖS methodologisch abgrenzen lässt. Die Implikationen dieser methodologischen Abgrenzung, und die daraus abgeleiteten „Selbstbeschränkungen der ÖS,“ missversteht Belke.

Im kurzen Abschnitt zur empirischen Analyse des Kontrafaktums verweist Belke auf zwei Arbeiten, in denen mit der sogenannten synthetic counterfactuals method (auch sysnthetic control method) die Kosten der Wiedervereinigung für Westdeutschland (Abadie et al. 2015), und die ökonomischen Vorzüge der Mitgliedschaft in der Europäischen Union für verschiedene Länder geschätzt werden (Campos et al. 2014). Dabei wird die tatsächlich beobachtbare historische Entwicklung einer makroökonomischen Kernvariable, wie etwa dem BIP pro Kopf, mit einem konstruierten Kontrafaktum verglichen. Dieses wird über einen gewichteten Durchschnitt von Daten aus Kontrollländern berechnet. Es ist aber völlig unklar auf welcher Basis Belke, oder die zitierten Verfechter dieser Methode, das angewandte Schätzverfahren für „robust“ erklären können.

Die Robustheit eines statistischen Schätzers, als eine Eigenschaft in der theoretischen Statistik, ergibt sich daraus, dass Ausreißer in den beobachteten Daten bei der Berechnung des Schätzers heruntergewichtet oder gar ganz außer Acht gelassen werden. Um dies umsetzen zu können, muss entweder Wissen über die zugrundeliegende Wahrscheinlichkeitsverteilung vorausgesetzt werden, oder es muss ein pragmatisches Auswahlkriterium postuliert werden, nach dem sich die Gewichtung der Kontrollgrößen aus der Güte einer empirischen Prognose ergibt. Im Fall von Campos et al. (2014) wird die Gewichtung etwa derart gewählt, dass die durchschnittliche quadratische Abweichung zwischen dem konstruierten synthetischen Kontrafaktum und den tatsächlich beobachteten Daten für den jeweiligen Zeitraum vor Eintritt in die EU minimiert wird. ${ }^{6}$

Das synthetische Kontrafaktum und die tatsächlichen Beobachtungen liegen also typischerweise ganz dicht beieinander für den Zeitraum bevor das ,treatment“ (Eintritt in die EU) erfolgt. Wir finden also gerade in den von Belke zitierten Studien das von uns hervorgehobene methodologische Leitmotiv wieder: Das synthetische Kontrafaktum wird über den empirischen fit gerechtfertigt, bzw. damit, wie gut es die tatsächlich beobachteten Werte über den historischen Zeitraum vor Eintritt in die EU prognostizieren kann. Dabei sind in der zitierten Studie keinerlei Erwägungen zum Problem der Überanpassung (overfit) zu finden.

\footnotetext{
5 Es gibt mittlerweile sogar Bestrebungen die DSGE-Modelle in das Grundstudium zu integrieren (SolisGarcia 2018), wobei hier nach wie vor die IS-LM und AS-AD-Modelle die Oberhand behalten.

6 Dies führt zum Beispiel dazu, dass sich das kontrafaktische BIP-Wachstum für Spanien in dieser Studie im Wesentlichen aus einem gewichteten Durchschnitt der beobachteten Kontrollgrößen für Neuseeland $(35,8 \%)$, Brasilien $(37,3 \%)$ und Kanada (26,8\%) ergibt (Campos et al. 2014, S. 14).
} 
Die gesamte Glaubwürdigkeit der Analyse steht und fällt also mit der Annahme, dass das konstruierte synthetische Kontrafaktum annähernd akkurat ist, dass also die Spezifizierung des ökonometrischen Modells ${ }^{7}$ zur Beschreibung des Kontrafaktums geeignet ist und dies auch über die Zeit nach dem treatment bleibt. ${ }^{8}$ Gerade diese Annahme lässt sich aber nicht testen und bleibt letztendlich ein Glaubensakt.

Die auf Basis dieses ökonometrischen Verfahrens postulierten quantitativen Kausalzusammenhänge zwischen wirtschaftlicher Integration und BIP-Wachstum kommen nicht zwangsläufig einer verhängnisvollen Anmaßung gleich (Hayek 1992). Gleichwohl sind sie eine Anmaßung von Wissen. Sie spiegeln eine gewisse Gleichgültigkeit gegenüber der Komplexität ökonomischer Phänomene wider. Wer nicht an die zugrundeliegenden Annahmen glaubt, könnte derartige Studien auch unter der Kategorie „Eleganter Unsinn“ archivieren (Sokal und Bricmont 1999).

Belke scheint zu meinen, dass die Ökonometrie hilfreich bei der Erklärung der Vergangenheit ist und man nur dann übers Ziel hinausschießt, wenn man sie auch für Vorhersagen der Zukunft verwenden will. Aber diese Vorstellung ist ein Trugbild. Ökonometrie ist grundsätzlich ungeeignet als Werkzeug der Kausalforschung (Hoppe 1983). Das betrifft die zukünftige genau wie die vergangene Wirklichkeit. Ihr Dienst ist rein deskriptiv. Sie wirft Fragen auf, aber sie antwortet nicht. Insofern die empirische Forschung aus ökonometrischen Zusammenhängen Ursache und Wirkung ablesen will, ist sie eben nicht empirisch. Sie verlässt vielmehr den Boden der Wissenschaft und setzt Postulate an die Stelle von Tatsachen (Mises 1985). Daher ist sie auch ungeeignet zur Begründung wirtschaftspolitischer Maßnahmen, die sich ja immer auf Kausalzusammenhänge stützen müssen.

Ganz anders die theoretisch-qualitative Analyse im Sinne der klassischen Ökonomik oder der ÖS. Indem sie universelle Kausalzusammenhänge ans Licht bringt, kann sie bestimmten praktischen Empfehlungen als Grundlage dienen, völlig unabhängig von den besonderen zeitlichen und örtlichen Umständen. Die Vorzüge wirtschaftlicher Integration etwa, ergeben sich aus ganz grundsätzlichen Erwägungen: die Ausweitung des gemeinsamen Marktes und des Freihandels, die Intensivierung der Arbeitsteilung und Spezialisierung, die Möglichkeiten einer effizienteren Allokation von Kapital und Arbeit. Diese Zusammenhänge galten zu Zeiten der Klassiker wie auch heute, wenngleich sie nach wie vor nicht einwandfrei quantifiziert und auf eindimensionale Kerngrößen heruntergebrochen werden können.

In einem anderen Abschnitt fragt Belke nach unserer Positionierung zu einem ,augenfälligen und öffentlichkeitswirksamen Problem." Es geht um die offensichtlich falschen empirischen Prognosen mancher „Praktiker der ÖS“ im Hinblick auf Preisinflationsentwicklungen. Diese Fehlgriffe stellen aus unserer Sicht kein größeres Problem dar als etwa die falschen Prognosen von „Mainstream“-Ökonomen. Wer sich wider die grundsätzlichen Prinzipien der ÖS zu derartigen Prognosen hinreisen lässt, sollte in jedem Fall auch bereit sein, eines Besseren belehrt zu werden. Es

\footnotetext{
${ }^{7}$ Dies schließt sowohl die funktionale Form des Modells, als auch die Auswahl der erklärenden Variablen und der potentiellen Kontrollländer mit ein.

${ }^{8}$ Für den Zeitraum vor dem Eintritt in die EU sind Kontrafaktum und Faktum identisch. Daher kann die Güte der Schätzung des Kontrafaktums durch seine Nähe zum beobachteten Faktum für diesen Zeitraum evaluiert werden.
} 
bleibt festzuhalten, dass Kritik an Blogposts einiger Autoren weder notwendig, noch hinreichend für fundierte Kritik an der ÖS ist. Wenn eine Brücke zusammenbricht, weil ein Ingenieur falsch gerechnet hat, werden auch nicht gleich Mathematik und Physik in Frage gestellt.

Belke legt es den Vertretern der Österreichischen Schule nahe, sich nicht gegen den wissenschaftlichen Wettbewerb zu immunisieren und selbst „,mehr Mut bei der zielführenden Anwendung ökonometrischer Methoden und der Heranziehung von Ergebnissen stringenter ökonometrischer Studien [zu] offenbaren." Dem kann an sich nicht widersprochen werden. Die Frage ist, was genau sich hinter dieser Empfehlung verbirgt. Wenn Belke darunter versteht, dass die ÖS ihre, nach unserem Dafürhalten, gut begründeten methodologischen Prinzipien aufgeben sollte, so scheint spätestens hier ein Punkt erreicht, an dem sich ein ganz bedeutender Unterschied zwischen ÖS und „Mainsteam“ offenbart, und zwar unabhängig davon, ob es sich um den interventionskritischen oder -freundlichen Teil des letzteren handelt. Hier kann letztlich nur der fortlaufende Austausch und die Abwägung von Argumenten zielführend sein. Die Vertreter der ÖS (wie auch der SÖ) können sich gegen Kritik nur insofern und nur dadurch ,immunisieren“, dass sie die besseren Argumente hervorbringen; und sie sollten auch kritisiert werden, wann immer ihnen dies nicht gelingt.

Nun könnte es vielleicht aus strategischer Sicht sinnvoll sein, sich der Methodik des Mainstreams zu bedienen, um eine größere Durchschlagskraft in der öffentlichen und politischen Debatte zu erwirken. Ohne Zweifel würde dies, den Fortbestand der ÖS als wissenschaftlich seriöse Denkschule gefährden. Doch das wäre keinesfalls der einzige Schaden, der aus dieser Taktik entstünde. Die Wahl der Methode aus politischen Gründen wäre vielmehr ein Bärendienst an der Wissenschaft im Allgemeinen und sicherlich am von Belke gelobten Wettstreit der besseren Ideen. Strategische Erwägungen sind der Wissenschaft eben fremd. Man mag sie schlitzohrig heranziehen, aber wäre damit auch schon auf abschüssigem Grund. Wenn sie wider besseren Wissens herangezogen werden, hat man den wissenschaftlichen Geist im Grunde schon über Bord geworfen. In Anspielung auf einen alten Witz - und es sei uns gestattet, hiermit zu schließen - lässt sich sagen: Ein echter Österreicher kann nicht gleichzeitig intelligent und ehrlich und ein traditioneller Ökonometriker sein.

\section{Literatur}

Abadie, A., Diamond, A., \& Hainmueller, J. (2015). Comparative politics and the synthetic control method. American Journal of Political Science, 59(2), 495-510.

Bachmann, R. (2019). Erfolge und Probleme der modernen (Mainstream-)Makroökonomik. List Forum für Wirtschafts- und Finanzpolitik. https://doi.org/10.1007/s41025-019-00121-y

Basse, T. (2006). An Austrian version of the Lucas critique. The Quarterly Journal of Austrian Economics, 9(1), 15-26.

Campos, N. F., Coricelli, F., \& Moretti, L. (2014). Economic growth and political integration: estimating the benefits from membership in the European union using the synthetic counterfactuals method. IZA DP No. 8162.

Cruccolini, R. (2010). Primat Der Form: Zur Methodologie Und Geschichte in Der Modernen Makroökonomik. München: Universität München. Dissertation an der Volkswirtschaftlichen Fakultät der Ludwig Maximilians Universität

Friedman, M. (1953). The methodology of positive economics. In Essays in positive economics (S. 3-43). Chicago and London: University of Chicago Press. 
Hausman, D. M. (2008). The philosophy of economics—an anthology (3. Aufl.). Cambridge: Cambridge University Press.

von Hayek, F. A. (1992). The fatal conceit: the errors of socialism. London: Routledge.

Hoppe, H.-H. (1983). Kritik der kausalwissenschafilichen Sozialforschung - Untersuchungen zur Grundlegung von Soziologie und Ökonomie. Opladen: Westdeutscher Verlag.

Israel, K.-F. (2015). Modern monetary policy evaluation and the Lucas critique. Political Dialogues: Journal of Political Theory, 19, 123-145.

Krugman, P. (2009). How did Economists get it so Wrong? New York Times Magazine September.

Lindé, J. (2018). DSGE models: still useful in policy analysis? Oxford Review of Economic Policy, 34(1-2), 269-286.

Lucas, R.E. (1976). Econometric policy evaluation: a critique. In K. Brunner \& A. H. Meltzer (Hrsg.), The Phillips curve and labor markets: carnegie-rochester conference series on public policy (S. 19-46). Amsterdam: North-Holland.

Lucas, R.E. (1981). Studies in business cycle theory. Cambridge: MIT Press.

von Mises, L. (1985). Theory and history: an interpretation of social and economic evolution. Auburn: Ludwig von Mises Institute.

Romer, P. (2016). The trouble with macroeconomics. Commons Memorial Lecture of the Omicron Delta Epsilon Society.

Snowdon, B., \& Vane, H. R. (1998). Transforming macroeconomics: an interview with Robert E. Lucas Jr. Journal of Economic Methodology, 5(1), 115-146.

Sokal, A., \& Bricmont, J. (1999). Eleganter Unsinn. Wie die Denker der Postmoderne die Wissenschaften mißbrauchen. München: C.H.Beck.

Solis-Garcia, M. (2018). Yes we Can! Teaching DSGE models to undergraduate students. The Journal of Economic Education, 49(3), 226-236. https://doi.org/10.1080/00220485.2018.1464987

Stiglitz, J.E. (2011). Rethinking macroeconomics: what failed, and how to repair it. Journal of the European Economic Association, 9(4), 591-645.

Stiglitz, J.E. (2017). Where modern macroeconomics went wrong. National Bureau of Economic Research Working Paper Series. 